\title{
Electrostatic Latching for Inter-module Adhesion, Power Transfer, and Communication in Modular Robots
}

\author{
M. E. Karagozler, J. D. Campbell, G. K. Fedder, S. C. Goldstein, M. P. Weller, B. W. Yoon.
}

\begin{abstract}
A simple and robust inter-module latch is possibly the most important component of a modular robotic system. This paper describes a latch based on electric fields and capacitive coupling. Our design provides not only significant adhesion forces, but can also be used for inter-module power transmission and communication. The key insight presented in this paper, and the factor that enables electrostatic adhesion to be effective at the macroscale, is the use of electric field attraction to generate frictional shear forces rather than electric field attraction alone. A second important insight is that a specific degree of flexibility in the electrodes is essential to maximize their mutual coupling and the resulting forceselectrodes which are too flexible or too rigid will perform less well. To evaluate the effectiveness of our latch we incorporate it into a cubic module $28 \mathrm{~cm}$ on a side. The result is a latch which requires almost zero static power and yet can hold $0.6 \mathrm{~N} / \mathrm{cm}^{2}$ of latch area.
\end{abstract}

\section{INTRODUCTION}

Modular robotics offers the tempting prospect of robotic systems which can be simultaneously cost-effective, robust, and yet flexible. Since early work on CEBOT [3] there have been numerous research projects aimed at realizing this promise (see [10] for a recent survey). Despite progress made in the past 15 years, creating a robust modular robotic system still poses significant engineering challenges. One key challenge is the realization of a strong, lightweight, robust, manufacturable inter-module latching mechanism. In this paper we describe a novel modular robot latch based on electrostatic attraction. Our latch design is suitable for lattice style robots at both the micro- and macroscale.

An ideal inter-module latch would provide structural stability, power distribution, and a communication channel between adjacent robots. It would also be self-aligning, genderless, quick to engage/disengage with minimal insertion/removal force required, weigh little with respect to the rest of the robot, and consume no power to maintain a latched connection. To date, latch design has required significant compromises with respect to these ideals. Latches are often heavy, take up significant amounts of space, require many seconds to engage/disengage, or provide only some of the desired capabilities. For example, the latch on the ATRON robot is one of the sturdiest implemented [10]. However, it

This work was supported in part by NSF Grants CNS-0428738, ITR0326054 and Intel Corporation.

Karagozler, Fedder, and Yoon at Electrical and Computer Engineering at Carnegie Mellon University, 5000 Forbes Ave, Pittsburgh, PA 15213 \{mkaragoz, fedder, byoon\} @ece. cmu. edu. Campbell at Intel Research Pittsburgh \{jason.d.campbell\}@intel.com. Goldstein in Computer Science Department at Carnegie Mellon University sethecs. cmu . edu. Weller in Architecture Department at Carnegie Mellon University philetus ecmu . edu.

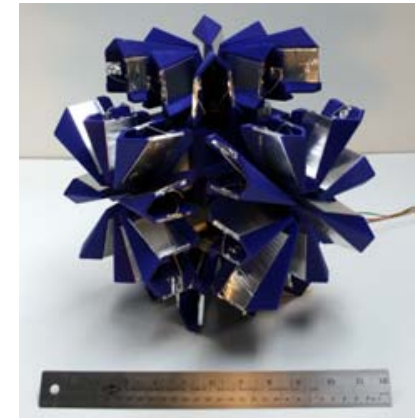

(a) A fully contracted unit.
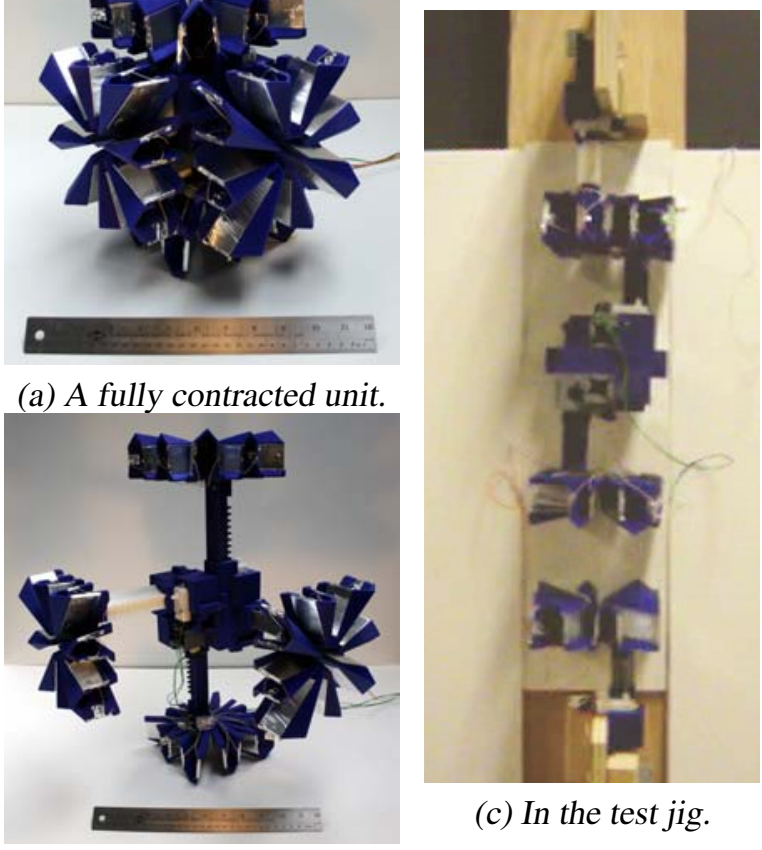

(c) In the test jig.

(b) A fully extended unit.

Fig. 1. Electrostatic-based modular robots. Related pictures and video can be found at www.cs.cmu.edu/ claytronics/iros07-latch.html

accounts for well over half of each module's volume and weight.

In the past, latches for centimeter-scale robots have been based upon either mechanical engagement (e.g., [10], [7], [12], [15], [11]) or magnetic forces (e.g., [14], [9], [8], [6]). Electric field adhesion has been studied only for systems at millimeter-scale and below. In 2006, we experimented with electrostatic latches for a short-lived robot that we called a "giant helium catom" (GHC) [5]. The GHC was an $8 m^{3}$ cube filled with helium to offset most of its weight and emulate the high surface area to weight ratios likely for submillimeter-scale robot modules. The GHC latching mechanism was based upon electrostatic attraction between the faces of two adjacent cubes. While in theory the expected forces (given the large area of the faces) should have been sufficient to latch two robots together, the mechanism failed due to peeling (see Section II). This failure led us to a new latch design that harnesses shear forces rather than normal forces. This change in force orientation eliminated the fatal peeling found in the GHC mechanism. Our current prototype of this new design can generate holding force of more than 


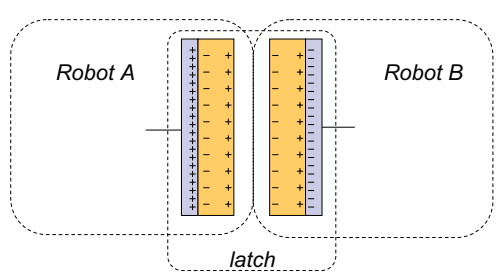

Fig. 2. Electrodes are aligned, forming the latch.

$0.6 \mathrm{~N} / \mathrm{cm}^{2}$ of latch area, when actuated with a voltage of $500 \mathrm{~V}$.

When one mechanism can serve two or more purposes, the total weight, volume, and complexity requirements for a module may be reduced, potentially allowing higher performance and improved scaling. The design of an electrostatic latch is simple, yet multipurpose: each robot has two halves of a capacitor on its face, and when faces from neighboring robots come in contact the pair of capacitors is complete. Viewing the latch as a pair of capacitors naturally leads to the idea of using these capacitors as a power and communication link between the robots. We detail our investigations in power transfer in Section III.

In this paper we demonstrate an electrostatic latch on a prototype modular robot modeled after the Crystalline Atom [11] and TeleCube [14]. The modules attach in a cubic lattice using electrostatic latches, and move their faces via expanding arms nested in the central frame, as shown in Fig. 1a and b. This modular robot design is covered in more detail in Section IV.

\section{An EleCtrostatic LATCH}

An ideal modular robotic latch should:

- have a high adhesion force when locked,

- lock and unlock easily,

- be self aligning,

- use minimal power during locking and unlocking,

- use no power once locked.

One approach to electrically latching two robot modules would be to use electrostatic forces between parallel capacitor plates. If the plates are closely spaced, then charging the capacitor will generate an electrostatic force attracting the plates of the capacitor to one another. Neglecting leakage, residual charge will then keep the latch closed indefinitely. (Thus the latch would use no power to remain attached.) The latching force may subsequently be released by discharging the capacitor.

Such a latch is illustrated in Fig. 2. For electrical insulation, the conductive plates are separated by a thin dielectric film on each of them. For practical systems there will also be some intervening gap between the electrodes. The capacitance and the electrostatic force for the parallel plate capacitor are:

$$
C=\frac{A \epsilon_{0}}{\left(l+2 d / \epsilon_{r}\right)}, \quad F=\frac{A \epsilon_{0} V^{2}}{2\left(l+2 d / \epsilon_{r}\right)^{2}}
$$

where $A$ is the surface area of the electrodes, $V$ is the applied voltage, $d$ is the thickness of the dielectric coating,

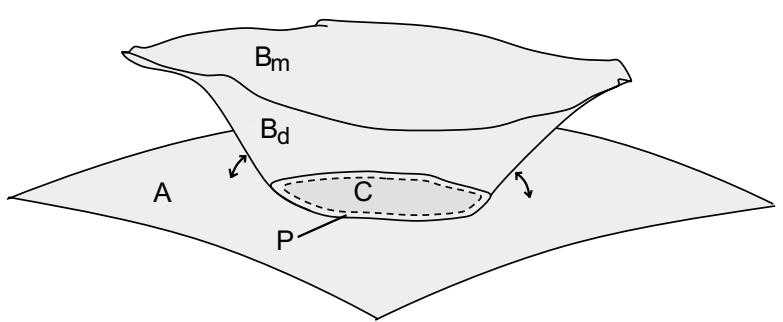

Fig. 3. Two flexible electrodes conforming onto each other: A: Bottom electrode, $B_{m, d}=$ Top electrode metal and dielectric side, $C$ : Contact area, $P$ : Boundary of contact.

$l$ is the separation between electrodes, $\epsilon_{0}$ in the dielectric permittivity of vacuum and $\epsilon_{r}$ is the relative permittivity of the dielectric coating.

Equation 1 dictates that for tens of Newtons of electrostatic force over an area of hundreds of $\mathrm{cm}^{2}$, the separation between the electrodes must be on the order of microns. Because of the exponential relationship between capacitance and inter-electrode distance, any additional separation (even just a few microns) will significantly reduce the generated force. Thus, when the electrodes are made out of rigid conductors (e.g., thick aluminum plates), even small levels of surface asperity (roughness) or dirt prevent the required close engagement. Rigid structures at this scale simply can not be made flat enough to be practical. Mechanical compliance, or flexibility of the electrodes offers a way to achieve closer spacing and obtain a large electrostatic force.

When flexible electrodes are charged, even if the initial separation is large, the (much smaller) electrostatic force developed can cause the electrodes to bend and more closely approach one another, reducing the separation and allowing larger electrostatic forces to be created. As a result of this feedback mechanism, the flexible electrodes move towards each other until they completely conform to each other resulting in significant adhesion forces.

However, flexible electrodes suffer from an effect we call peeling (see Fig. 3), which limits the amount of adhesion that can be sustained. Flexibility means that portions of the electrodes' surfaces can be separated without (much) impact on the separation of the rest of the surface (see region $\mathrm{P}$ in Fig. 3). Working incrementally, even a small force can then detach one electrode from another much like unzipping a zipper.

By reorienting the primary applied force in the latch to run along the surface of each electrode rather than normal to it, we can eliminate the potential for peeling. In the shear direction a force must act on the entire surface area at once. Careful design of electrode geometries can maximize the use of shear force and minimize the use of normal force.

Our latch design is based on this realization about shear forces. The contact surface of the electrodes is parallel to the direction of latch engagement. Thus, when the latch engages and locks, the shear force created by the electrostatic adhesion holds the latch together. 


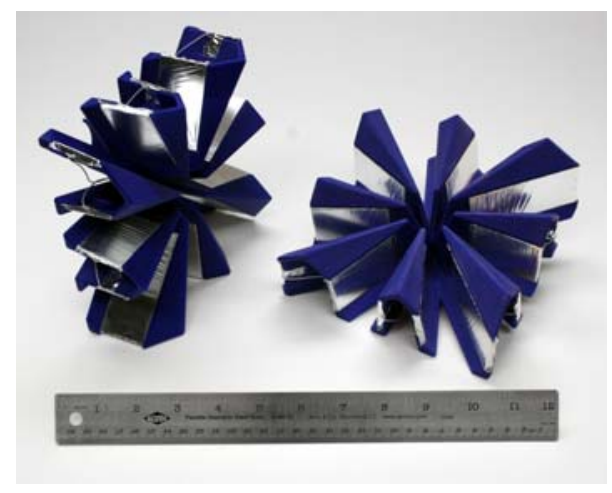

Fig. 4. A latch, composed of two faces.

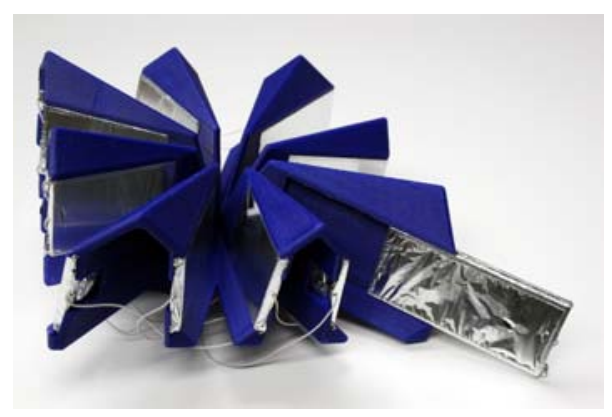

Fig. 5. The electrodes slide into slots on the star shaped face.

\section{A. Implementation of a Macroscale Electrostatic Latch}

Each latch is composed of two genderless "faces" (Fig. 4). A face is a star shaped plastic frame to which the electrodes are attached. Each face carries multiple electrodes, and each electrode is made from a thin aluminum foil coated with dielectric film wrapped and glued onto a plastic panel. The completed electrodes (panel and film) then slide into the corrugated star shaped face as shown in Fig. 5. The face has both the normal surfaces necessary to create shear forces, and also gives the latch the ability to self-align. As the faces engage (Fig. 6), the faces are guided both translationally and rotationally into position by the face shape, correcting any initial misalignment.

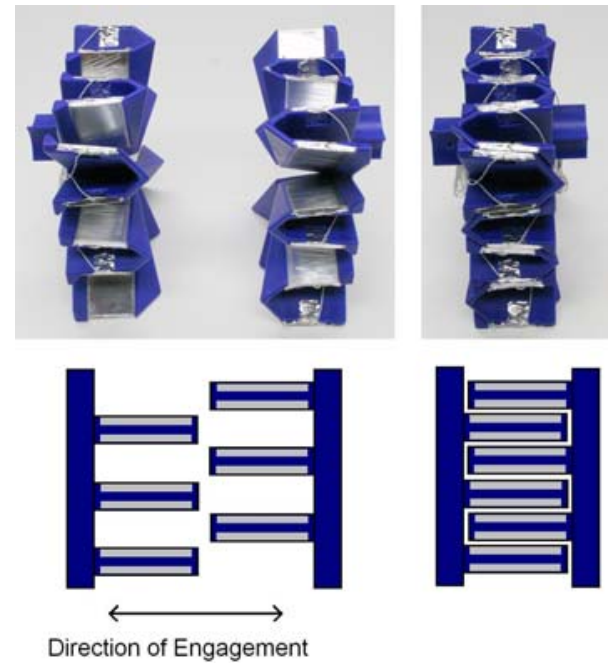

Fig. 6. The two faces of the latch engaging.
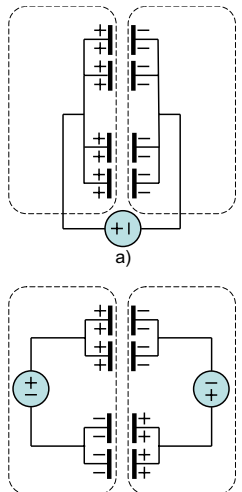
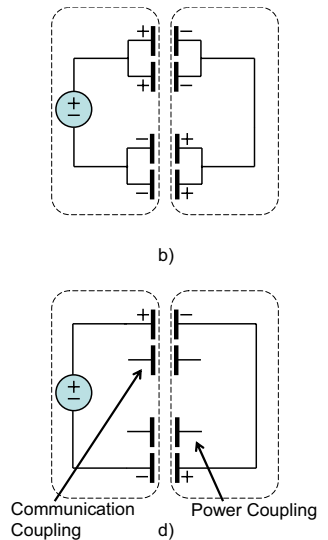

Fig. 7. Various methods to excite latches.

The latch is locked when a voltage is applied between the electrodes of the two faces. However, since each face of the latch is located on a different robot, the electrodes cannot share a common ground as in Fig. 7a. Having multiple electrodes allows for various alternative ways to excite the latch. As shown in Fig. 7b, the latch can be excited by applying a voltage difference on electrodes of only one face, while the other face is passive. Fig. 7c shows another connection diagram where there is a voltage source on both faces, effectively increasing the voltage drop on each individual electrode pair by a factor of two. This increases the generated force by a factor of four. Also, as explained in Section III, some of the electrodes can be dedicated to power transfer or communication, as shown in Fig. 7d.

Our current latch prototype is $16 \mathrm{~cm} \times 16 \mathrm{~cm} \times 6 \mathrm{~cm}$ in overall dimensions. The aluminum foil used is $20 \mathrm{~nm}$ thick, and the dielectric (mylar) coating of the foil is $6 \mu \mathrm{m}$ thick. From equation 1, with $500 \mathrm{~V}$ applied to the electrodes, the normal force generated can be calculated as:

$$
F_{\text {normal }}=\frac{\left(283 \times 10^{-4}\right)\left(8.85 \times 10^{-12}\right)(500)^{2}}{2\left(0+2\left(6 \times 10^{-6}\right) / 3.2\right)^{2}}=2255 \mathrm{~N}
$$

This calculation assumes that the electrodes conform to each other completely and there is no separation between them. In general, to get the maximum force, we would like to increase the voltage up to just below the level at which dielectric breakdown occurs.

The shear force due to friction, which is the force the latch can withstand before disengaging, can be calculated as:

$$
F_{\text {shear }}=F_{\text {normal }} * \mu_{\text {mylar-on-mylar }}=451 N
$$

Here, $\mu_{\text {mylar-on-mylar }}=0.2$ [1].

Unlocking the latch is achieved by draining the charge on the faces of the adjacent robots. Our experiments have shown that over time, as the latch is engaged and disengaged again and again, a small fixed charge can accumulate on the dielectric. Even after the charge across adjacent pairs of electrodes have been drained, residual charge is present due 


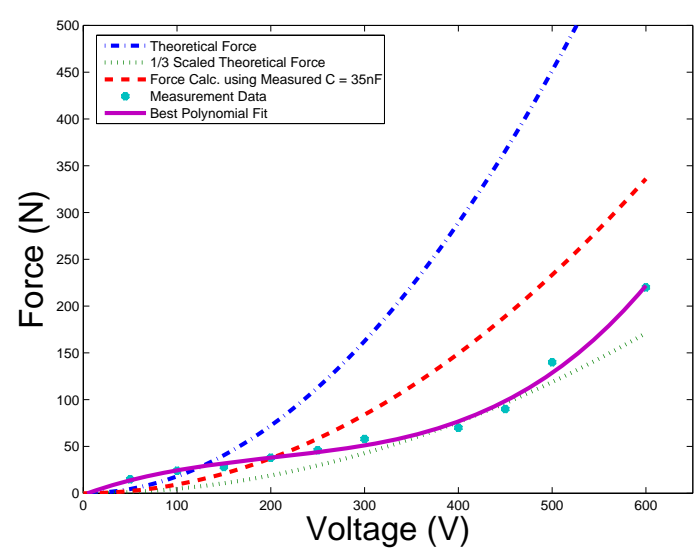

Fig. 8. Latch force versus applied voltage.

to trapped space charge [2]. This is particularly true when, as in our case, the dielectric has inherent defects. Since the dielectric has a high resistance, the charge trapped in the defects of the material remains even after the electrodes has been drained. If unaccounted for this residual charge requires some force to separate the latch. This can be solved in two ways. First, dielectrics without defects (e.g., oxide based thin films) can be used. Second, the polarity of the electrodes can be switched every time the latch is engaged. By reversing the polarity the trapped charge can be more effectively drained out of the dielectric.

To ease latch disengagement, each electrode surface is designed to have a slight release angle. This release angle introduces a small amount of the peeling effect mentioned above, and as a result, although a high force is maintained when the electrodes are charged, the latch disengages easily-even if there is residual charge present after draining the capacitor.

\section{B. Evaluation of the Macroscale Electrostatic Latch}

In order to verify the electrostatic force, force measurements were taken with varying voltages applied to the electrodes. At each voltage, the latch was forced apart with an increasing load until disengagement, and the amount of load that caused disengagement was recorded. Fig. 8 compares the theoretical and measured latch force.

Experimental data shows that at $500 \mathrm{~V}$, the measured force is significantly lower than the theoretical value, by a factor of three. Moreover, the measured force graph grows slower than $F=k V^{2}$ at smaller voltages, but starts to grow faster as the voltage reaches $\approx 400 \mathrm{~V}$. This is due to the assumption in the theoretical force calculation that there is no separation between electrodes, i.e., the analysis assumes perfect electrode conformance. However, the electrode conformance is directly related to the force generated and thus directly to the applied voltage. We see that the electrodes do not conform well when the applied voltage is below $400 \mathrm{~V}$. Once the applied voltage reaches $400 \mathrm{~V}$, the electrodes almost completely conform and one can see that the measured force begins to "catch up" to the predicted force.

Although the measured force is in the range of hundreds of Newtons and is suitable for macroscale modular robotics applications, it is still significantly lower than the theoretical value. This is due to several factors:

- The release angle designed to ease latch disengagement introduces the highly nonlinear peeling effect, which causes a decrease in the adhesion force. We observed reduced force for bigger release angles, however, having no release angle causes difficulty in latch disengagement.

- As trapped charge builds up over time, the polarity of excitation is switched to get rid of the trapped charge. However, some amount of trapped charge is always present, which reduces repeatability of experiments and introduces significant measurement error.

- We observed that as the electrodes conform, due to the high electrostatic pressure, wrinkles form on the foils, thus, the effective area in equation 1 is less than the actual electrode area.

- The analysis assumes that the separation $L$ between electrodes is zero; this might not be the case as there is no simple way to tell whether air is trapped in between electrodes or if the observed wrinkles cause separation.

- Experiments revealed that the characteristics of the latch change with how tightly the foil is attached to the panel. When it is too tight the foil on the panel cannot conform to the adjacent plate.

The capacitance between the electrodes of two faces was also measured, when the latch is engaged. As predicted, the capacitance measured when the electrodes are charged (with $500 \mathrm{~V})$ is significantly higher $(C \cong 35 n F)$ then when they are uncharged, but in contact with each other $(C \cong 8 n F)$. The measured capacitance is also lower than the theoretical value of $C=68 n F$ by a factor of $\approx 2$. This phenomena arises from the last four factors described above. (The first of the factors, the release angle, is strictly related to the force generated, not the capacitance.)

\section{Electrostatic Latches at the Microscale}

In this section we discuss the feasibility of using similarly designed latches at smaller scales (i.e. micrometers). The main question involved in using this design at smaller size scales is whether the conforming property of the flexible foil still exists, since this property is what actually creates the adhesion. To determine whether the material and electrostatic properties at the macroscale still hold in the microscale, we investigated a model as shown in Fig. 9. It is assumed that two square plate electrodes having the dimensions shown are charged with voltage $V$ at the macroscale. For the MEMS scale model, we scale the dimensions equally with a linear factor $\alpha$ and scale the voltage separately with factor $\kappa$, where $\alpha<1$ and $\kappa<1$.

To simplify the analysis we assume that only one electrode is flexible and conforming, while the other is rigid. Also, the dielectric coating and the metal layer are modeled with a single layer plate. These assumptions are both conservative and reasonable-experiments at the macroscale show that the latch is effective even when only one electrode is 


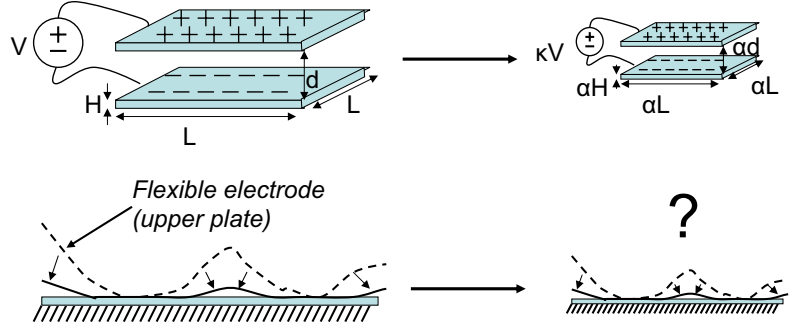

Fig. 9. Scaling down electrostatic latching.

flexible, e.g., a rigid aluminum sheet and a flexible foil-mylar electrode create significant shear forces.

The square plate that is rigidly attached at all sides bends in the presence of uniform pressure $P$ according to equation 4 [13]:

$$
\begin{array}{r}
P=\left\{\frac{3 \pi^{2}}{2}\left[\frac{\sigma_{0} H}{L^{2}}\right]+\frac{2 \pi^{4}}{3}\left[\frac{E H^{3}}{\left(1-v^{2}\right) L^{4}}\right]\right\} w+ \\
\frac{\pi^{4}(7-2 v)(5+4 v)}{128(1+v)}\left[\frac{E H}{(1-v) L^{4}}\right] w^{3}
\end{array}
$$

Here, $w$ is a geometrical term that represents the deflection of the center of the plate. $E$ is the Young's modulus of the material, $\sigma_{0}$ is the residual stress that is created due to the manufacturing process, $v$ is the poisson's ratio, $H$ is the thickness of the plate, $L$ is the side length of the square plate.

For our analysis, $w$ represents how much the plate bends, which is also the measure of how much the plate conforms. Rather than the exact solution, we are mainly interested in the dependency of $w$ on dimensions and voltage, so with only a small loss in accuracy we will consider just the cubic $\left(w^{3}\right)$ term in Equation 4. In the model described above, the pressure is the electrostatic force per unit area, with the simplifying assumption that it is uniform and does not change as the plate bends. Then, considering only the second term in equation 5 , the resulting dependency is:

$$
\begin{gathered}
P=\frac{F}{L^{2}}=K \frac{H}{L^{4}} w^{3}, F=F_{\text {electrostatic }}=\frac{1}{2} \frac{A V^{2}}{d^{2}} \\
\frac{1}{L^{2}} \frac{1}{2} \frac{A V^{2}}{d^{2}}=K \frac{H}{L^{4}} w^{3} \\
w^{3}=K^{\prime} \frac{L^{4}}{H} \frac{V^{2}}{d^{2}}
\end{gathered}
$$

At the MEMS scale, we assume all dimensions are scaled with $\alpha$ and voltage is scaled with $\kappa$. Then, we have:

$$
w_{\text {scaled }}^{3}=\frac{\alpha^{4} L^{4}}{\alpha H} \frac{\kappa^{2} V^{2}}{\alpha^{2} d^{2}}=\alpha^{3} w^{3} \frac{\kappa^{2}}{\alpha^{2}}=\alpha \kappa^{2} w^{3}
$$

Ideally, we would like to have $w_{\text {scaled }}=\alpha w$, since we would like to have the MEMS scale plate bend the same way as the macro scale plate, which will ensure similar conformance at the at the MEMS scale.

To have $w_{\text {scaled }}=\alpha w$, the condition is $\kappa=\alpha$. This implies that to have the electrostatic latching work the same way it does at the macro scale, if all the dimensions are scaled down with a factor, the applied voltage must also be scaled down with the same factor. If this condition is satisfied, the bending happens in similar proportion to the macroscale version, confirming in principle that the electrostatic latching mechanism scales.

Finally, scaling the applied voltage with the same factor also ensures that the magnitude of the electric field created $|E|=V / d$ stays constant, which is crucial in preventing dielectric breakdown.

In addition to the assumptions made above, MEMS scale latches require the consideration of additional factors. MEMS thin film materials behave very differently from their macroscale counterparts. Thus designing appropriate latch behavior requires the use of non-traditional values for properties such as Poisson's ratio, Young's modulus, and permittivity. Furthermore, environmental factors such as dust require much greater attention at smaller scales.

\section{Summary}

The measured adhesion force our prototype panels generates is $0.6 \mathrm{~N} / \mathrm{cm}^{2}$ of face area at $500 \mathrm{~V}$. This corresponds to $0.53 \mathrm{~N} / \mathrm{cm}^{2}$ of electrode area. Since the electrode is normal to the plane of the face this gives the latch designer an additional parameter to work with when designing a face. The electrodes are versatile in their arrangement on the latch and can be made self-aligning. The energy needed to lock and unlock the latches is very low-the theoretical value is $W=8.5 \mathrm{~mJ}$. This is the amount of energy required to lock the latch once. The latch consumes no power once locked, and the stored energy may even be partially recovered.

In short, the latch is strong, power efficient and self aligning. In addition, the analysis reveals that the same idea can be applied to smaller scale latches. Making use of shear forces, the latch generates forces high enough for macroscale modular robotics applications. In Section IV a modular robot that integrates the electrostatic latches is discussed.

\section{POWER TRANSFER}

In this section we describe how to harness capacitive coupling between adjacent modules to transfer power through an ensemble. Much as a transformer uses an electromagnetic coupling between coils to transmit power without a direct electrical contact, a pair of capacitors can be used to carry energy across a non-conducting gap. A potential advantage of capacitive transfer is that it should not require the high mass coils in a transformer or the high contact forces typically needed for DC connectors to break through oxide layers, counteract surface roughness, or operate in dirty environments.

The following analysis demonstrates the potential for power transport between adjacent modules via electrostatic coupling using $\mathrm{AC}$ excitations. Fig. 10 illustrates the general approach used. Note that neither a common ground nor any non-capacitively-coupled connections between modules are required.

Fig. 11 shows the diagram of the circuit representing the model that is analyzed. We use a square wave generator as 


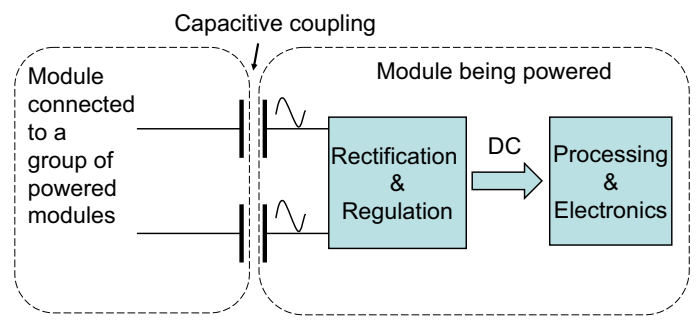

Fig. 10. Power transfer between modules.

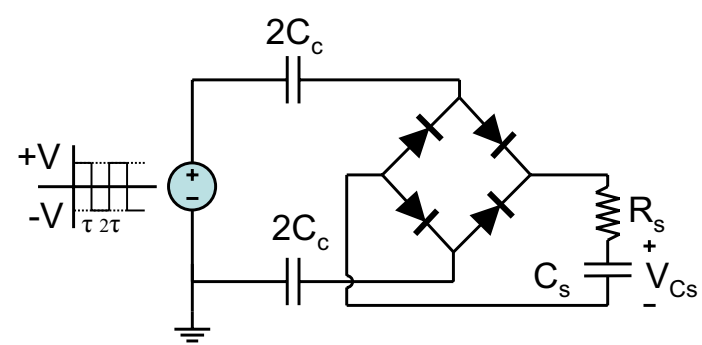

Fig. 11. A model for power transfer between robots; $2 C_{c}$ 's denote the coupling capacitors while $C_{s}$ denotes the storage capacitor.

the voltage source to simplify the analysis. This source is coupled to the target module through two capacitors, each of value $2 C_{c}$. On the target module, there is a full wave rectifier built out of 4 diodes, and the module's storage capacitor $C_{s}$. Also, there is a resistance $R_{s}$ that represents the lumped series resistance of the circuit.

The two most important parameters that characterize the power transfer are the charge time; $t_{C H A R G E}$, the time required to reach a certain voltage level at the target module and the power transfer efficiency; $E$, the ratio of the power transferred over the power consumed. While $t_{C H A R G E}$ determines how fast an ensemble can be powered, $E$ practically determines the number of modules that can be powered. Assuming that each module that receives power from one of it's neighbors is to transfer power to another neighbor, the power requirements grow exponentially with increasing number of modules, thus making it infeasible to power many modules if the efficiency is low.

After each transition of the source voltage, the storage capacitor is charged to a value dictated by the ratio of the storage and coupling capacitors. Eventually, the voltage reaches a final value, assuming that the time constant of the $R C$ circuit is much smaller compared to the period of $\mathrm{AC}$ excitation, $2 \tau$.

After $n$ transitions, the voltage on $C_{s}, V_{C_{s} n}$, where $n \in Z^{+}$denotes the number of source voltage transitions, is (details of this derivation can be found in [4], [16]):

$V_{C_{s} n}=V\left[1-\left(\frac{C_{s}-C_{c}}{C_{c}+C_{s}}\right)^{n-1}\right]+V_{C_{s} 1}\left(\frac{C_{s}-C_{c}}{C_{c}+C_{s}}\right)^{n-1}$

An important property of this relation is that as time passes, the storage capacitor voltage $V_{C_{s} n}$ reaches $V$, that is, $\lim _{n \rightarrow \infty} V_{C_{s} n}=V$. Therefore, it can be concluded that any voltage level up to the source voltage can be achieved a)

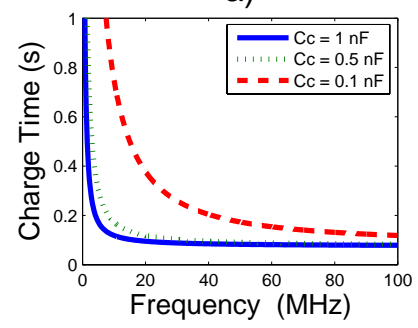

b)

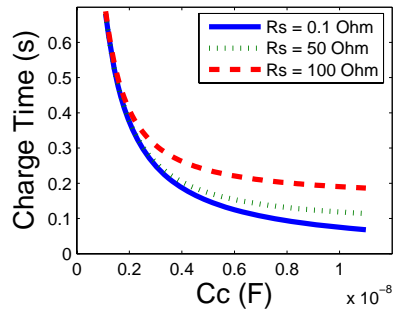

Fig. 12. a: Charge time versus frequency $f$ of input square wave, $b$ : Charge time versus coupling capacitance $C_{c}$. Unless specified: $C_{c}=1 \times 10^{-9} \mathrm{~F}$, $C_{s}=1 \times 10^{-3} F, R_{s}=50 \Omega, f=1 M H z, \kappa=0.95$.

at the target capacitor, provided that $n$ is large enough; given enough time. For the case where $C_{s}=1 \mathrm{mF}$ and $C_{c}=10 n F$, to reach $95 \%$ of the source voltage $V$, the number of transitions required is $n=150,000$. For a source excitation frequency of $1 M H z$, the target voltage is reached in $t_{C H A R G E}=75 \mathrm{~ms}$.

This analysis suggests that to further decrease the charge time the source frequency must be increased. To obtain a more precise relationship requires us to model the finite lumped series resistance $R_{s}$ which results in a more precise version of Equation 9 as:

$$
\begin{aligned}
& V_{C_{s} n}=V-V\left(\frac{C_{s}-C_{c}+2 C_{c} e^{\frac{-\left(C_{s}+C_{c}\right)}{R_{s} C_{c} C_{s}} \tau}}{C_{c}+C_{s}}\right)^{n-1} \times \\
&\left(1-\frac{C_{s}}{C_{c}+C_{s}}\left(1-e^{\frac{-\left(C_{s}+C_{c}\right)}{R_{s} C_{c} C_{s}} \tau}\right)\right)
\end{aligned}
$$

From the above we can solve for $t_{C H A R G E}$ which is plotted in Fig. 12. The preceding analysis shows that the charge time $t_{C H A R G E}$ decreases asymptotically as the frequency $f$ or the coupling capacitance $C_{c}$ is increased. In fact, it can be shown that:

$$
\lim _{f \rightarrow \infty} t_{C H A R G E}=\frac{1}{2} R_{s} C_{s} \ln \left(\frac{C_{s}}{\left(C_{c}+C_{s}\right)(1-\kappa)}\right)
$$

Equation 11 shows that $t_{C H A R G E}$ decreases for smaller values of $R_{s}$. For better (faster) power transfer between modules, the coupling capacitance and the excitation frequency should be maximized while the lumped series resistance is kept minimal.

Another very important measure is the power transfer efficiency, $E$. When enough time passes, all the energy is stored on the storage capacitor $C_{s}, W_{C_{s}}=\frac{1}{2} C_{s} V_{C_{s}}^{2}$, and no energy is stored on coupling capacitor $C_{c}$, since $\lim _{n \rightarrow \infty} V_{C_{c} n}=0$. However, to calculate the efficiency, the energy that is dissipated on the series resistance $R_{s}, W_{R_{s}}$, must be found. Then,

$$
E=\frac{W_{\text {Stored }}}{W_{\text {Total }}}=\frac{W_{C_{s}}}{W_{C_{s}}+W_{R_{s}}}
$$

To find $W_{R_{s}}$, the series resistance voltage $V_{R_{s}}$ must be found. Using 9 and $V_{R_{s}}(t)=R_{s} C_{s} \frac{d V_{C_{s} n}}{d t}$, we can obtain 
expressions for $V_{R_{s}}(t)$ after $n$ transitions and then derive the power and energy dissipation from the $n$th to $(n+1)$ th transitions. The energy dissipated on $R_{s}$ as $n \rightarrow \infty$ (or equivalently $t \rightarrow \infty$ ), and the efficiency $E$ is:

$$
\lim _{n \rightarrow \infty} W_{R_{s}}=\frac{1}{2} V^{2} \frac{C_{s}^{2}}{\left(C_{c}+C_{s}\right)}, \quad E=\frac{C_{c}+C_{s}}{C_{c}+2 C_{s}}
$$

It must be noted that the efficiency is bounded by $2 / 3$, as this is the case where $C_{c}=C_{s}$, and the analysis is not valid for $C_{c}>C_{s}$. For typical values of $C_{c}$ and $C_{s}$ given in Section II, $E \cong 0.50$. Also, the efficiency is independent of $R_{s}$.

\section{A. Summary}

Using the electrodes on each face of the latch to transfer power between modules is an appealing solution, but our results to date demonstrate low efficiency which makes this method infeasible for large ensembles. Other methods that depend on capacitive coupling but utilize different circuitry and potentially achieve higher efficiencies should be investigated.

Capacitive data transfer between adjacent modules is even simpler than power transfer, and it should be possible to achieve both data and power transfer utilizing the same coupling electrodes as latching.

\section{Demonstration Cubic Robot}

To demonstrate the ideas presented in the previous sections we designed and built a cubic lattice-style modular robot which uses electrostatic latching. The robot has a packing and gait similar to Rus and Vona's Crystalline Atom [11] and PARC's Telecube [14]. These modules reconfigure using an inchworm gait-by expanding the connected faces of two neighboring modules so that one of them is pushed one block length across the assembly, and then contracting to pull the next module forwards. (Fig. 1c shows a module in the middle of such a movement, and a video of latch operation is at WwW. CS.cmu.edu/ claytronics/iros07-latch.html.)

Cubic shapes have several desirable properties: 1) the positions of modules are highly constrained by the cubic lattice which simplifies planning and mating; 2) Rus and Vona [11] have demonstrated that with a sufficiently large metamodule any arbitrary configuration of voxels is reachable; and 3) modules can propagate through the center of an assembly allowing the system to reconfigure more quickly than systems where modules can only move along surfaces of an assembly.

Because our latch provides adhesion, power transfer and communication in a simple and robust package, it reduces the complexity of the entire system. By reducing the number of systems in each module we simplify fitting them within the available space, and enhance their manufacturablity. This makes it feasible to produce larger numbers of modules and experiment with more interesting behaviors.

\section{A. Actuation Subsystems}

Our modules are composed of two active systems: an electrostatic latch that provides adhesion, and a modular worm drive assembly that expands and contracts the faces using a DC-motor. We use passive systems to provide alignment and to limit the extension of the faces to avoid the need for any further sensing or actuation components.

1) Mechanical Actuation for Expansion and Contraction: The linear actuation subsystem drives arms attached to each face so that the opposing faces of the module can move from a fully contracted state $(22 \mathrm{~cm}$ between faces) to to a fully expanded state ( $44 \mathrm{~cm}$ between faces). Each face has its own dedicated, independently controlled worm drive housed in a modular tube. The arm attached to each face fits into this tube. The six tubes containing the face assemblies are bolted together to create the central frame for the robot as shown in Fig. $1 \mathrm{~b}$.

2) Genderless Self-aligning Comb Latch: Although we could have used male and female mating latches, we used a genderless latch to reduce the number of components in the system and ease assembly. As shown in Fig. 4, each face of the robot is corrugated in a radial comb. The top of each comb terminates in a forty-five degree blade to provide passive self-alignment. The vertical faces of the combs have slots for the electrodes. Two faces mate tightly together to provide a rigid moment connection. The vertical surfaces of the combs have a five degree release angle to allow the faces to retract easily once the charge has been drained from the electrodes.

The two most important constraints involving the design of faces are total electrode area and depth. Electrode area determines the amount of force generated, whereas the depth of each face must be small enough to let the face easily clear neighboring latches when actuated. Each face has sixteen $3 \mathrm{~cm}$ deep capacitive panels mounted on eight combs. We could have designed the latch with fewer larger combs which would increase the amount of misalignment that could be passively corrected, however, this would increase the height of the blades and make it more difficult to achieve a compact unit overall. We choose to reduce the depth of the plates to permit a more compact packing of the modules.

\section{B. Impact of Latch on Overall Design}

As with most robot designs, cubic lattice-style modular robots must balance a variety of sometimes conflicting design goals relating both to the performance of individual parts, and of the system as a whole. Here we describe how the features of our latch impact the design of the entire system:

Robust Docking: Other systems have used either mechanical or magnetic latches to connect and disconnect from neighboring modules. Both of these strategies have problems. Mechanical latches such as Polybot's [15] and ATRON's [10] tend to seize and fail to disengage under load unless they use very large, heavy motors relative to the size of the module. Switching permanent magnet latches such as the TeleCube's [14] provide a limited amount of force, are heavy and require complex mechanical actuators. Our electrostatic 
latch provides a robust, lightweight electronically actuated mechanism for both latching to and unlatching from neighboring modules. When activated it provides a great deal of force, and when deactivated the two neighboring faces separate easily with little force.

As our electrostatic latch also can be used for power transfer and communication, it allows us to reduce module complexity in comparison to other systems which need separate subsystems to handle each of these functions. Our latch design also lends itself to being inexpensively mass produced as it consists of simply a plastic frame, an an array of electrodes, and the wiring to connect them.

Passive Self-alignment: Although the rigid packing of modules within a crystalline lattice helps to align modules there is inevitably some degree of misalignment that must be corrected when two modules mate. The shape of our latches forces the combs into alignment as they move closer together. This passive self-aligning mechanism is able to correct misalignments of up to $18 \%$ of the width of a face, or a rotational misalignment of up to 40 degrees. We believe that this is a significant improvement over other systems and one of the strongest points of our design.

This robust passive self-alignment mechanism eliminates the need for extra sensing components to detect when modules are near enough to actuate the latch. The arms of the neighboring modules are simply driven towards each other until a current sensor on the motor power line indicates that there is mechanical resistance, and then the electrodes are charged to lock the faces together.

Reducing System Weight: Our electrostatic latching system greatly reduces the overall weight of our modules compared to mechanical and permanent magnet latches, and can also allow for a better latch-to-total weight ratio. When we compare the weight of six faces and the electronics for driving the electrodes with the weight of the entire module we find our latch takes less than $40 \%$ of the total. This fraction would be even smaller if our latch components were refabricated via injection molding or machining rather than our present finite-deposition-modeling parts. In comparison, an ATRON module's latches account for $60 \%$ of the weight of the entire module.

\section{Summary}

We evaluated the latch design using $3.5 \mathrm{~kg}$ robot modules. As required by the robot's inchworm gait, these modules were able to robustly lock onto each other with sufficient rigidity to translate one module three body lengths. Our latch provided self-alignment and easy engagement, and as one module released another the latch easily disengaged. Finally, the latch proved strong enough to pull another module along.

\section{Conclusions}

The electrostatic latch we describe in this paper offers robust adhesion, self-alignment, fast engagement, and easy detachment, all at a reduced weight and lower energy budget when compared to other latch designs. Despite strong latching force it remains very easy to disengage. The key insight that makes this combination of properties possible is that electric-fields can be harnessed to generate significant frictional shear forces between flexible, capacitively coupled electrodes. Use of shear forces rather than direct electric field attraction avoids the negative effects of peeling, while still retaining the tolerance to initial air gaps and dust particles provided by flexible electrodes. Our capacitively coupled latch also has the ability to function as the basis for intermodule communication and power transfer (albeit at low efficiencies to date). We also presented a cubic robot module design that illustrates the reduction in complexity made possible by our electrostatic latch.

\section{REFERENCES}

[1] http://www.goodfellow.com/csp/active/static/e/polyethylene_ terephthalate.html.

[2] L. Dissado, G. Mazzanti, and G. C. Montanari. The role of trapped space charges in the electrical aging of insulating materials. IEEE Transactions on Dielectrics and Electrical Insulation, 4(5):496-506, 1997.

[3] Toshio Fukuda, Tsuyoshi Ueyama, Yoshio Kawauchi, and Fumihito Arai. Concept of cellular robotic system (cebot) and basic strategies for its realization. Comput. Electr. Eng., 18(1):11-39, 1992.

[4] Mustafa Emre Karagozler. Harnessing capacitance for inter-robot latching, communication, and power transfer. In MSc Report, Carnegie Mellon University, Department of Electrical and Computer Engineering, May 2007.

[5] Mustafa Emre Karagozler, Brian Kirby, W.J. Lee, Eugene Marinelli, T.C. Ng, Michael Weller, and Seth Copen Goldstein. Ultralight modular robotic building blocks for the rapid deployment of planetary outposts. In Revolutionary Aerospace Systems Concepts Academic Linkage (RASC-AL) Forum 2006, Cape Canaveral, FL, May 2006.

[6] Brian Kirby, Burak Aksak, James F. Hoburg, Todd C. Mowry, and Padmanabhan Pillai. A modular robotic system using magnetic force effectors. In In Proceedings of the IEEE International Conference on Intelligent Robots and Systems (IROS '07), October 2007.

[7] K. Kotay, D. Rus, M. Vona, and C. McGray. The self-reconfiguring robotic molecule. In IEEE International Conference on Robotics and Automation (ICRA), pages 424-31, 1998.

[8] S. Murata, H. Kurokawa, and S. Kokaji. Self-assembling machine. In Proc. IEEE Int. Conf. Robotics and Automation, pages 441-8, 1994.

[9] Satoshi Murata, Eiichi Yoshida, Akiya Kamimura, Haruhisa Kurokawa, Kohji Tomita, and Shigeru Kokaji. M-tran: Selfreconfigurable modular robotic system. IEEE/ASME Transactions on Mechatronics, 7(4):431 - 441, December 2002.

[10] Esben Hallundbaek Ostergaard, Kristian Kassow, Richard Beck, and Henrik Hautop Lund. Design of the atron lattice-based selfreconfigurable robot. Auton. Robots, 21(2):165-183, 2006.

[11] Daniela Rus and Marsette Vona. Crystalline robots: Selfreconfiguration with compressible unit modules. Autonomous Robots, 10(1):107-124, 2001.

[12] Behnam Salemi, Mark Moll, and Wei-Min Shen. SUPERBOT: A deployable, multi-functional, and modular self-reconfigurable robotic system. In iros-06, Beijing, China, oct 2006. To appear.

[13] Stephen D. Senturia. In Microsystem Design, pages 255-258. Kluwer Academic Publishers, 2002.

[14] J.W. Suh, S.B. Homans, and M. Yim. Telecubes: mechanical design of a module for self-reconfigurable robotics. In Proc. of the IEEE Int'l Conference on Robotics and Automation (ICRA), volume 4, pages 4095-101, 2002.

[15] M. Yim, D.G. Duff, and K.D. Roufas. Polybot: a modular reconfigurable robot. In IEEE International Conference on Robotics and Automation (ICRA), volume 1, pages 514-20, 2000.

[16] Byung Woo Yoon. Power delivery circuit for scalable claytronics. In MSc Report, Carnegie Mellon University, Department of Electrical and Computer Engineering, May 2007. 